\title{
Assessing the Performance Degradation of Lithium-Ion Batteries Using an Approach Based on Fusion of Multiple Feature Parameters
}

\author{
Anchen Wang, ${ }^{1}$ Ying Zhang $\mathbb{D}^{1,2}$ and Hongfu Zuo ${ }^{2}$ \\ ${ }^{1}$ College of Automobile and Traffic Engineering, Nanjing Forestry University, Nanjing 210037, China \\ ${ }^{2}$ College of Civil Aviation, Nanjing University of Aeronautics and Astronautics, Nanjing 210037, China
}

Correspondence should be addressed to Ying Zhang; zhangyingrms@163.com

Anchen Wang and Ying Zhang contributed equally to this work.

Received 28 January 2019; Revised 17 April 2019; Accepted 2 May 2019; Published 19 May 2019

Academic Editor: Konstantinos Karamanos

Copyright (c) 2019 Anchen Wang et al. This is an open access article distributed under the Creative Commons Attribution License, which permits unrestricted use, distribution, and reproduction in any medium, provided the original work is properly cited.

\begin{abstract}
A method based on fusion of multiple features is proposed to assess and accurately describe the performance degradation of lithiumion batteries in this paper. First, the discharge voltage signal of lithium-ion batteries under real-time monitoring is analyzed from the perspective of time domain and complexity to obtain the values of multiple features. Then, the multi-feature parameters undergo a spectral regression process to reduce the number of dimensions and to eliminate redundancy, and on the basis of this regression, a Gaussian mixture model is established to model the health state of batteries. Thus, the degree of lithium-ion battery performance degradation can be quantitatively assessed using the Bayesian inference-based distance metric. A case calculation experiment is carried out to verify the effectiveness of the method proposed in this paper. The experimental results demonstrate that, compared with other assessment methods, the performance degradation assessment method proposed in this paper can be used to monitor the degradation process of lithium-ion batteries more effectively and to improve the accuracy of condition monitoring of batteries, thereby providing powerful support for making maintenance decisions.
\end{abstract}

\section{Introduction}

As a kind of ideal power supply energy, which can be reused, lithium-ion battery has been widely used in many fields. However, the performance of lithium battery will decline over time, which will not only lead to system failure, but also lead to serious safety accidents [1]. Working condition monitoring, performance degradation assessment, and remaining useful life (RUL) prediction of lithium-ion battery are of great significance for improving the safety and reliability of the battery system and preventing battery accidents. The RUL prediction of lithium-ion battery can be divided into two steps: battery health status recognition and performance prediction. In terms of prediction model, Zou [2] used parameter degradation model and traceless particle filter algorithm to predict RUL of battery, discovering that the method for predicting the RUL of the battery was more accurate when considering the degradation of the performance parameters of the battery capacity state. Ma [3] proposed an improved trackless Kalman filter based on Markov chain-Monte Carlo to predict RUL, and an empirical model of four-parameter power function reflecting battery capacity was established. Linear least square method was used to estimate capacity parameters, and finally life prediction was realized with high prediction accuracy. Yang [4] used two logarithmic empirical capacity models to capture twophase nonlinear sag degradation problem of lithium-ion batteries, and an improved particle filter algorithm was used to update the model's state parameters to predict the RUL. The prediction model has achieved good results, but mostly used the battery capacity or single parameter as a feature to predict. However, the battery capacity is difficult to directly measure online, and a single parameter cannot describe the performance degradation of battery. Therefore, 
it is advantageous to accurately reflect the state of battery performance and improve the prediction accuracy of residual life by extracting characteristic parameters from multiple perspectives and fusing them into a health index, and this paper focuses on performance degradation assessment of lithium batteries.

To avoid problems such as insufficient maintenance or over-maintenance of lithium-ion batteries, it is necessary to carry out condition-based maintenance for batteries. The quantitative assessment of the degree of performance degradation is a prerequisite for condition-based maintenance of lithium-ion batteries. It is difficult to measure the residual capacity of batteries in real time [5], many researchers try to assess the performance of the battery using the approach of analyzing variations in the discharge voltage. Sbarufatti proposed a method that combines particle filters and radial basis function neural networks to predict the discharge duration of lithium-ion batteries [6]. Xu proposed an effective model based on state space for modeling the performance degradation of batteries. The model adopts an analysis method based on the sequentially observed discharging profiles and uses the expectation-maximization (EM) algorithm in conjunction with the extended Kalman filter (EKF) algorithm to estimate and update the model parameters and state [7]. Xu also analyzed the distribution of discharge voltage and combined the discharge process and the degradation process to develop a hierarchical model for comprehensive prediction of discharge duration in different cycles and the remaining effective cycles [8]. Widodo used the sample entropy of the discharge voltage to predict the performance of the battery [9].

Although some of the methods proposed in the above studies can be used to make effective assessment, they still fall short of the expectation of accurately and comprehensively assessing the degradation process of the batteries. This is because most of these methods only process a single feature of the discharge voltage, which is not very sensitive to variations in the battery state. To solve this challenge, this paper proposes a method based on the fusion of multiple features for assessing the performance degradation of lithium-ion batteries. In fact, the multi-feature fusion approach has been widely used in assessing the performance of bearings, but relatively fewer attempts have been made to adopt this approach for assessing the performance of batteries.

In researching the performance degradation assessment, effectively extracting multi-dimensional features and establishing an effective degradation assessment model are two critical issues. A lot of work has been done with regard to the first critical issue. Principal component analysis (PCA) is one of the most widely used data compression algorithms. Dong used PCA to reduce the dimensions of the parameters in bearing degradation process, and they obtained good results [10]. Yu proposed a method based on local preserving projection (LPP) to reduce the dimensions of the multidimensional features in the time domain, frequency domain, and time-frequency domain. The method is applied to the performance degradation assessment of bearings and verified the feasibility of the method through experiments [11]. Cai proposed a spectral regression (SR) method, which is a new type of model, for supervised, unsupervised, and semisupervised regression. Using SR to reduce the dimensions makes it possible to solve the projection function using the regression framework, thereby circumventing the thorny issue of decomposing the features of the dense matrix [12]. With regard to the second critical issue, $\mathrm{Yu}$ proposed a method based on multiple two-layer sparse autoencoder neural networks to fuse the features and used experiment to verify the superiority of this method [13]. Yu applied the Gaussian mixture model (GMM) model to the health assessment of bearings and achieved quantitative analysis of performance degradation of the bearings [11]. Batista proposed an algorithm based on improved support vector machine (SVM), reducing the error rate in diagnosis [14]. Ai proposed a fusion method based on n-dimensional characteristic parameters distance [15]. Hassani proposed a fusion model utilizes zSlices-based representation of general type-2 fuzzy logic systems to combine different SVMs [16]. Zhang proposed an exergy-based fusion method to fuse multiple transient states with the information of multiple sensors obtained in state monitoring [17]. Shao proposed a feature fusion method based on the deep belief network (DBN), taking advantage of the excellent learning ability of DBN [18]. Zhou proposed an NCA-based feature extraction method to reduce dimensionality and used a coupled hidden Markov model based on multichannel data acquisition for fault diagnosis [19]. In addition, Wang [20] also summarized methods to evaluate the health indicators based on Markov model, self-organizing mapping, wavelet change, and fuzzy cmeans transformation. All of the above methods have yielded good assessment results.

On the basis of the above analysis, this paper proposes a performance degradation assessment method based on the fusion of multiple features. First, the discharge voltage signals of lithium-ion batteries under real-time monitoring are analyzed to obtain features in the dimensions of time domain and complexity. Then, the multiple features undergo an SR processing to reduce the number of dimensions and eliminate redundancy; based on these processes, GMM is established to model the health status of batteries. Thus, the degree of performance degradation of batteries can be quantitatively assessed using the Bayesian inference-based distance (BID) metric. The experimental results show that the assessment method proposed in this paper can be used to monitor the degradation process of lithium-ion batteries more effectively and to improve the accuracy of condition monitoring of batteries. To the best of our knowledge, single characteristic parameters are used in most studies of lithium battery status assessment. However, there is redundancy and difference among parameters, so the characterization of lithium battery state is not accurate enough. Therefore, in this paper, the characteristic parameters are extracted from the perspectives of statistical measurement and complexity; after that the extracted features are fused by the GMMBID model proposed in this paper. Compared with a single parameter, the health index can characterize the performance degradation degree of lithium battery more accurately. 


\section{Feature Extraction}

The capacity of battery is a parameter that can accurately evaluate the performance, but it is difficult to obtain directly, which can only be obtained in the laboratory or offline state. Therefore, it is necessary to select some parameters that can be monitored online to estimate the degradation state of lithium-ion batteries. During the charging process, the charging mode of the battery is generally fixed; that is, the charging voltage and current are also fixed. The discharge current depends on the system payload, which can affect the health of the battery, but cannot reflect the degradation of the health state of the battery. At present, most studies have found that the discharge voltage of the battery will tend to degrade with an increase in the number of discharge cycles [6-11, 21]. Therefore, this paper focuses on analyzing the easy-to-measure discharge voltage of lithium-ion batteries to make a more accurate assessment of the battery performance degradation.

2.1. Time Domain Features. The voltage values of all of the discharge cycles of a lithium-ion batteries can be regarded as a time series of voltage. It is necessary to analyze this time series to extract the features that reflect the statistical pattern of the time series. The most common method for analyzing a time series is time domain analysis. Charakopoulos A.K. [22] estimated mainly nonlinear measures such as mutual information combined with descriptive statistics measures, as well as some linear and nonlinear dynamic detectors such as Hurst exponent, detrended fluctuation analysis, and Hjorth parameters.

With the increase of charging and discharging times of lithium-ion battery, its internal resistance and energy loss increase, which shows that the discharge duration will be shorter and shorter after the battery is fully charged. Therefore, the article adopted the method of statistical measurement such as the mean and RMS values to describe the changing trend of battery discharge voltage. Also, regarding the trend of the battery voltage at the same time interval increasing with the number of charge and discharge cycles, fixed-interval voltage difference can be constructed to characterize the degree of battery performance degradation.

2.1.1. Statistical Measurement. There are some conventional time domain features based on statistical measurement (see Table 1).

2.1.2. Fixed-Interval Voltage Difference. When a series of discharge voltage values are obtained by sampling the discharge voltage at fixed intervals, the voltage discrepancy between two neighboring samples is called the fixed-interval voltage difference. As the battery is used successive times (that is, the number of discharge cycles of a lithium-ion battery increases), the battery ages continuously, which is reflected by the increasingly shorter discharge duration. During each discharge cycle, the discharge voltage of a battery decreases from a higher voltage value to a lower value.

Each fixed time interval is denoted as $t$, and the fixedinterval voltage difference in the $i$-th discharge cycle is
TABLE 1: Statistical measurement.

\begin{tabular}{lc}
\hline Feature & \multicolumn{1}{c}{ Equation } \\
Mean & $x_{\text {mean }}=\frac{\sum_{n=1}^{N} x(n)}{N}$ \\
$\begin{array}{l}\text { Root mean } \\
\text { square }\end{array}$ & $x_{r m s}=\sqrt{\frac{\sum_{n=1}^{N}(x(n))^{2}}{N}}$ \\
$\begin{array}{l}\text { Standard } \\
\text { deviation }\end{array}$ & $x_{\text {std }}=\sqrt{\frac{\sum_{n=1}^{N}\left(x(n)-x_{m}\right)^{2}}{N-1}}$ \\
Skewness & $x_{\text {ske }}=\frac{\sum_{n=1}^{N}\left(x(n)-x_{m}\right)^{3}}{(N-1) x_{s t d}^{3}}$ \\
Kurtosis & $x_{k u r}=\frac{\sum_{n=1}^{N}\left(x(n)-x_{m}\right)^{4}}{(N-1) x_{s t d}^{4}}$ \\
& $x(n)$ is the voltage value \\
\hline
\end{tabular}

$$
V_{i}=V_{t_{\max }}-V_{t_{\min }}, \quad i=1,2, \ldots, n
$$

where $V_{t}$ is the voltage value at the end of the time interval $t$ and $V_{t_{\min }}$ is the voltage value at the beginning of the time interval $t$. The sequence of fixed-interval voltage values extracted from the discharge cycles can then be expressed as

$$
V=\left\{V_{1}, V_{2}, \ldots, V_{n}\right\}
$$

where $n$ is the number of discharge cycles.

2.2. Complexity. Entropy is a way to describe the complexity of a time series or the degree of internal chaos. Originally a concept in physics, the concept of entropy gradually expanded with the development of information theory and has become an indicator of system instability. Entropy is now widely used in system state monitoring and signal analysis. At present, the various forms of entropy (such as information entropy, sample entropy, and approximate entropy) are used as the characteristic parameters for measuring complexity.

Derived from the approximate entropy, the sample entropy provides a better entropy representation in signal analysis. Compared with other metrics such as related dimensions, sample entropy is better suited for classifying complex systems, including characterization of the certainty and randomness of the time series of finite data points [9]. Sample entropy has been applied in bearing fault diagnosis [23-25]. Therefore, in this paper, we chose sample entropy as a useful complement to the characteristic parameters. The algorithm works in the following way:

(1) Assume that there is an $\mathrm{N}$-dimensional time series $u(1), u(2), \ldots, u(N)$ obtained via sampling at fixed time intervals.

(2) Define the associated parameters $m, r$ of the sample entropy where the real number $m$ is the length of the comparative vector and the real number $r$ is the metric of similarity.

(3) Reconstruct the $m$-dimensional vector $X(1), X(2)$, $\ldots, X(N-m+1)$ where $X(i)=[u(i), u(i+1), \ldots, u(i+m-1)]$. 
(4) For $1 \leq i \leq N-m+1$, count the number of vectors that satisfy the following condition:

$$
\begin{array}{r}
B_{i}^{m}(r) \\
=\frac{\text { number of } X(j) \text { such that } d[X(i), X(j)] \leq r}{N-m}, \\
i \neq j
\end{array}
$$

where $d[X(i), X(j)]$ is defined as

$$
\begin{aligned}
& d[X(i), X(j)]=\max _{a}\left|u(a)-u^{*}(a)\right|, \\
& X(i) \neq X(j)
\end{aligned}
$$

where $u(a)$ is an element of the vector $X, d$ is the distance between the vectors $X(i)$ and $X(j)$ is determined from the maximum difference of the corresponding elements, and the value range of $j$ is $[1, N-m+1](i \neq j)$.

(5) Calculate the average of $B_{i}^{m}(r)$ for all $i$ values, and denote the average value as $B^{m}(r)$, which can be expressed as

$$
B^{m}(r)=(N-m+1)^{-1} \sum_{i=1}^{N-m+1} B_{i}^{m}(r)
$$

(6) Let $k=m+1$, and repeat steps (3) and (4) to obtain

$$
A^{k}(r)=(N-k+1)^{-1} \sum_{i=1}^{N-k+1} A_{i}^{k}(r)
$$

where $A_{i}^{k}(r)=$ (number of $X(j)$ such that $d[X(i), X(j)] \leq$ $r) /(N-k), i \neq j$.

(7) The sample entropy is defined as

$$
\text { SampEn }=\lim _{n \rightarrow \infty}\left[-\ln \frac{A^{k}(r)}{B^{m}(r)}\right]
$$

In practical applications, $N$ will never be $\infty$. Therefore, when $N$ is a finite value, the sample entropy is estimated to be

$$
S a m p E n=-\ln \frac{A^{k}(r)}{B^{m}(r)}
$$

2.3. Spectral Regression. Given a data set $z_{1}, z_{2}, \ldots, z_{m} \in R^{n}$, the mapping element of $h_{1}, h_{2}, \ldots, h_{m} \in R^{l}(l<<n)$ can be found in the low dimension space for a given point $m$, and we can find a transformation matrix $A$ that satisfies $h_{i}=A^{T} z_{i}$.

A graph $G$ was constructed with $m$ samples using the idea of graph embedding, a process of mapping the data of a graph (usually a high dimensional dense matrix) into a low dimensional dense vector. Each vertex of the graph corresponds to a sample (the $i$-th point is $z_{i}$ ). If all of the weights of the connections between sample $i$ and sample $j$ are represented by a weight matrix $W$, then $W_{i j}$ is the weight of the connection between the $i$-th point and $j$-th point. The process of finding the linear projection function using the
SR method is divided into two steps: (1) Solve $W y=\lambda D y$, where $D$ is the diagonal matrix $D_{i j}=\sum_{j} W_{i j}$. The feature vector corresponding to the first $l$ feature value is defined as $y_{0}, y_{1}, \ldots, y_{l-1}$. (2) Obtain the projection vector $a$ by solving the following regularized least-square:

$$
a=\arg \min _{a}\left[\sum_{i=1}^{m}\left(\mathrm{a}^{T} z_{i}-y_{i}\right)^{2}+\lambda\|a\|^{2}\right]
$$

where $\lambda$ is the regularized parameter. The original data can then be dimension-reduced to an $l$-dimensional vector:

$$
z \longrightarrow h=A^{T} z, \quad A=\left(a_{0}, a_{1}, \ldots, a_{l-1}\right)
$$

\section{Performance Degradation Assessment Model Based on Fusion of Multiple Features}

Data fusion can be defined as a combination of multiple sources to obtain improved information; in this context, improved information means less expensive, higher quality, or more relevant information. The available data fusion techniques can be classified into three nonexclusive categories: (i) data association, (ii) state estimation, and (iii) decision fusion [26]. The data fusion method described in this paper is a state estimation fusion method.

3.1. GMM. GMM is a clustering algorithm that uses a Gaussian distribution as the parameter model and is trained using the EM algorithm. As a simple extension of the Gaussian model, GMM uses a combination of multiple Gaussian distributions to characterize the distribution of data, which is expressed as

$$
p(x)=\sum_{m=1}^{M} \pi_{m} p\left(x \mid \theta_{m}\right)
$$

where $M$ is the number of model mixing, $\pi_{m}$ is a weight coefficient of the model $\left(\sum \pi_{m}=1\right), p\left(x \mid \theta_{m}\right)$ is the Gaussian distribution function (the $m$-th average value is $\mu_{m}$ and the covariance matrix is $S_{m}$ ) denoted as $\phi=$ $\left\{\pi_{1}, \ldots, \pi_{m} ; \mu_{1}, \ldots, \mu_{m} ; S_{1}, \ldots, S_{m}\right\}$. The model can be rewritten in the following form:

$$
p(x \mid \phi)=\sum_{m=1}^{M} \pi_{m} p\left(x \mid \theta_{m}\right)
$$

After the GMM model was established using the data sets for the normal operation state, a quantitative indicator based on BID [27] was used to perform quantitative assessment of the degradation degree of lithium-ion batteries.

Suppose that there are $K$ Gaussian classes. The $k$-th component can be denoted as $C_{k}$, and its occurrence probability can be denoted as $\alpha_{k}$. The probability of the test point $x_{t}$ then belongs to the $k$ component $C_{k}$ (denoted as $p\left(C_{k} \mid x_{t}\right)$ ) and can be calculated using the following formula:

$$
p\left(C_{k} \mid x_{t}\right)=\frac{\alpha_{k} p\left(x_{t} \mid C_{k}\right)}{p\left(x_{t}\right)}=\frac{\alpha_{k} p\left(x_{t} \mid C_{k}\right)}{\sum_{i=1}^{K} \alpha_{i} p\left(x_{t} \mid C_{k}\right)}
$$




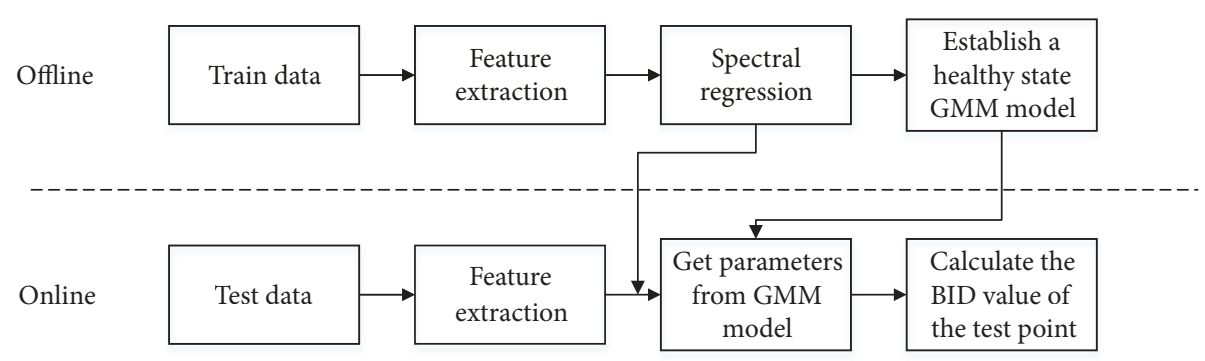

FIGURE 1: Flowchart of proposed performance degradation assessment method.

where $\alpha_{k}$ is the a priori probability that can be calculated using the model data. $p\left(x_{t} \mid C_{k}\right)$ can be calculated using the following formula:

$$
\begin{aligned}
p & \left(x_{t} \mid C_{k}\right) \\
& =\frac{1}{(2 \pi)^{1 / 2}\left|S_{k}\right|^{1 / 2}} \exp \left[-\frac{1}{2}\left(x_{t}-\mu_{k}\right)^{T} S_{k}^{-1}\left(x_{t}-\mu_{k}\right)\right]
\end{aligned}
$$

where $\mu_{k}$ is the average value of the $k$-th Gaussian component, $S_{k}$ is the covariance matrix of the $k$-th Gaussian component. Next, the distance from $x_{t}$ to each component $C_{k}$ is defined as

$$
D_{C_{k}}\left(x_{t}\right)=\left(x_{t}-\mu_{k}\right)^{T} S_{k}^{-1}\left(x_{t}-\mu_{k}\right)
$$

The BID value can be obtained by performing a weighted summation over all of the component distance values of a test point:

$$
B I D=\sum_{k=1}^{K} p\left(C_{k} \mid x_{t}\right) D_{C_{k}}\left(x_{t}\right)
$$

3.2. Performance Degradation Assessment Model Based on Fusion of Multiple Features. On the basis of the above analysis, this paper proposes a method based on the fusion of multiple features for assessing the performance of lithium-ion batteries. The flowchart of the method is shown in Figure 1.

Figure 1 shows that the performance degradation assessment process is divided into an offline phase and online phase. The specific steps of each phase are as follows:

(1) Off-Line Phase. (A) Extract initial features from the training data and use the SR method to reduce the dimensions of the original features.

(B) Select the health state training data to establish a GMM model and obtain the parameters of the model.

(C) Calculate the BID value of the test data using the GMM parameters to describe the performance degradation process of lithium-ion batteries.

(2) Online Phase. (A) Extract initial features from the training data and use the SR method to reduce the dimensions of the original features.

(B) Calculate the BID value between the test data and the health state GMM.

\section{Lithium-Ion Battery Performance Degradation Assessment Experiment and Results Analysis}

4.1. Data Source. A case calculation experiment was carried out to verify the reliability and effectiveness of the proposed method in assessing the performance degradation of lithiumion batteries. In the experiment, the experimental data sets were provided by NASA [28]. A set of four Li-ion batteries (\# 5, 6, 7, and 18) were run through 3 different operational profiles (charge, discharge, and impedance) at room temperature. Charging was carried out in a constant current (CC) mode at $1.5 \mathrm{~A}$ until the battery voltage reached $4.2 \mathrm{~V}$ and then continued in a constant voltage (CV) mode until the charge current dropped to $20 \mathrm{~mA}$. Discharge was carried out at a constant current (CC) level of $2 \mathrm{~A}$ until the battery voltage fell to $2.7 \mathrm{~V}, 2.5 \mathrm{~V}, 2.2 \mathrm{~V}$, and $2.5 \mathrm{~V}$ for batteries $5,6,7$, and 18 , respectively. Impedance measurement was carried out through an electrochemical impedance spectroscopy (EIS) frequency sweep from $0.1 \mathrm{~Hz}$ to $5 \mathrm{kHz}$. Repeated charge and discharge cycles result in accelerated aging of the batteries while impedance measurements provide insight into the internal battery parameters that change as aging progresses. The experiments were stopped when the batteries reached end-of-life (EOL) criteria, which was a $30 \%$ fade in rated capacity (from $2 \mathrm{Ahr}$ to $1.4 \mathrm{Ahr}$ ).

In order to save space, the batteries No. 5, No.6, and No. 18 of the four batteries are randomly selected for analysis. Figure 2 depicts the relationship between the discharge voltage and the discharge time of the three batteries. As seen in the figure, the different discharge cycles share a common pattern. In the early stage of discharge, the discharge voltage declines gradually, and the discharge time has a gentle slope. The slope of the decline increases gradually over time. In the last stage of discharge, the voltage drops rapidly. As the number of discharge cycles increases, the slope of the decline of the discharge voltage becomes increasingly steep. The variation of the discharge voltage in each discharge cycle differs from the voltage variation in any other discharge cycle; hence, it is necessary to assess and manage the battery.

4.2. Comparison of Clustering Ability Concerning Degradation Degree. In the experiment, 132 original data sets of battery number 18 are extracted. After a normalization process, 25 sets of normal state data, 25 sets of slight degradation data, and 25 sets of severe degradation data are used to verify 


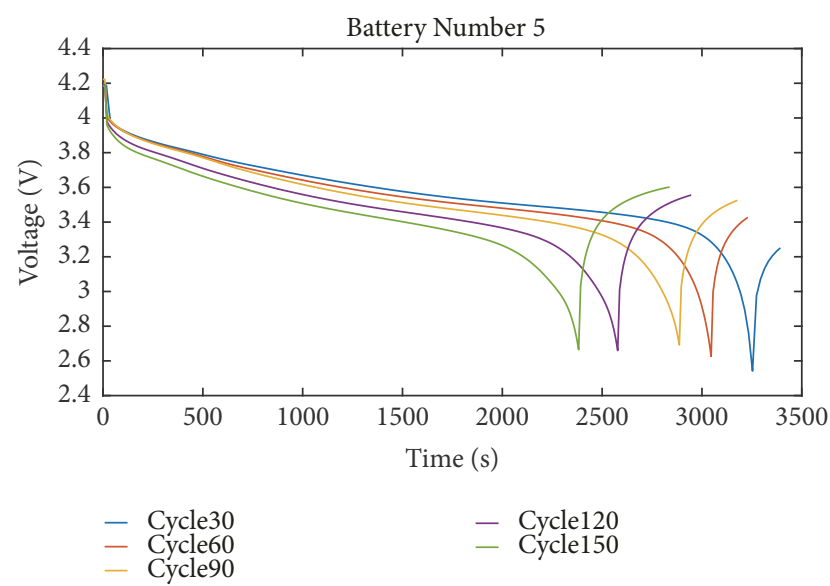

(a) Variation of discharge voltage of battery number 5

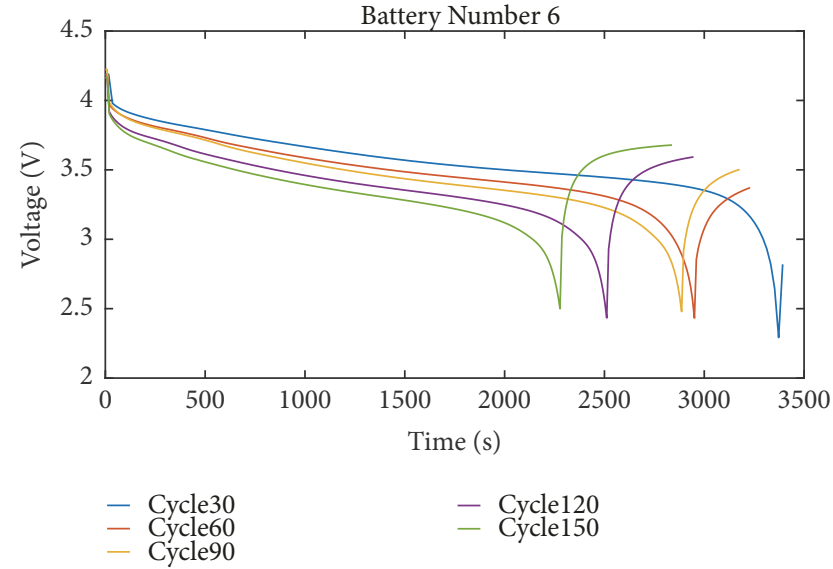

(b) Variation of discharge voltage of battery number 6

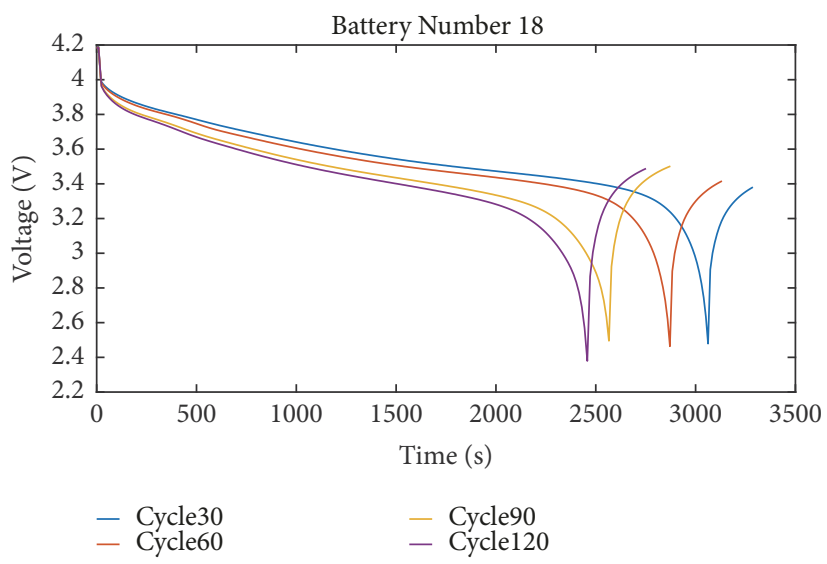

(c) Variation of discharge voltage of battery number 18

FIGURE 2: Variation of discharge voltage of battery numbers 5, 6, and 18.

the clustering ability and correctness of the SR dimension reduction method proposed in this paper. Furthermore, the SR algorithm is compared with the LPP and PCA algorithms. Figure 3 depicts the effects of the three algorithms in dimension reduction (only the first two dimensions are shown for each algorithm). To compare the three feature extraction methods more directly, the clustering algorithm $\mathrm{K}$-means is used as the degradation degree classifier to calculate the cluster recognition rates of the three methods. The results are shown in Table 2. Figure 3 shows that, after the dimension reduction, the PCA method failed to cluster the three states (i.e., the three states overlap with each other), which means that PCA has the worst clustering effect among the three algorithms. The LPP and SR algorithms have better clustering effects, as indicated by their ability to clearly distinguish the three states. Between these two algorithms, SR is superior to LPP. Table 2 also shows that PCA has the lowest cluster recognition rate among the three algorithms. The cluster recognition rate of SR is $97.33 \%$, which is $4 \%$ higher than that of the LPP algorithm. In summary, the SR dimension reduction algorithm has stronger clustering ability and recognition rate. Therefore, the SR method is more suitable for on-line monitoring.
TABLE 2: Clustering recognition rate of the three dimension reduction methods.

\begin{tabular}{lc}
\hline Dimension reduction algorithm & Recognition rate \\
\hline PCA & $90.67 \%$ \\
LPP & $93.33 \%$ \\
SR & $97.33 \%$ \\
\hline
\end{tabular}

4.3. Results of Performance Degradation Assessment. Seven representative features (namely root mean square (RMS), standard deviation, mean, skewness, kurtosis, fixed-interval voltage difference, and sample entropy) are used to assess the performance degradation of battery numbers 5, 6, and 18 using the existing data sets. After several trials, the number of the length of the comparative vector and the metric of similarity of sample entropy are set to $m=1, r=0.1$, respectively. The variation curves of the six features are shown in Figure 4. The following can be observed from the diagrams: For the same battery, different features exhibit different variation trends. In terms of overall trends, RMS, mean, and kurtosis tend to decline with fluctuations, whereas the standard deviation, skewness, fixed-interval voltage 


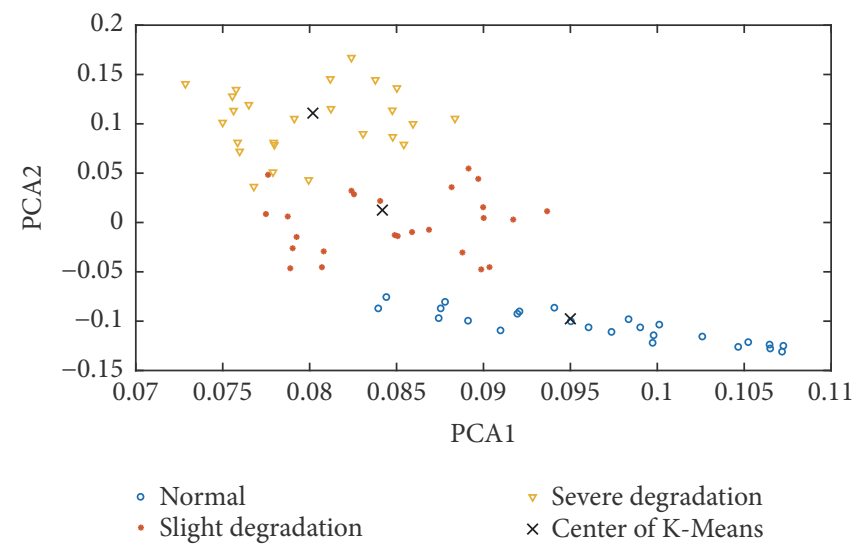

(a) Results of PCA-based clustering

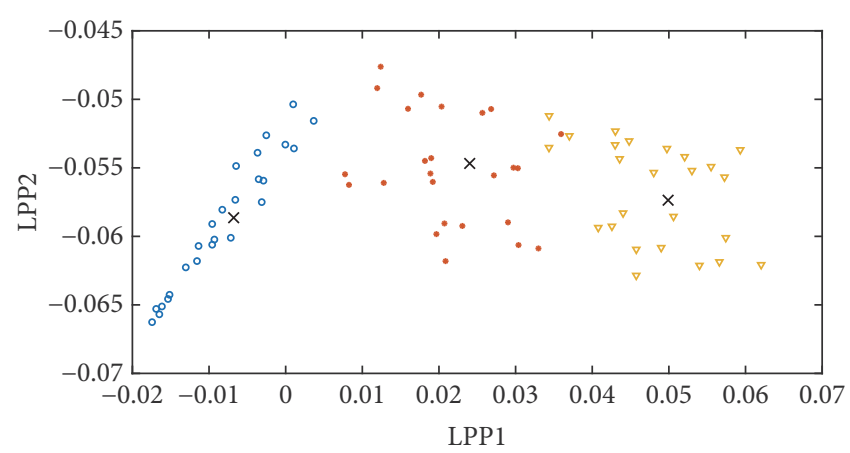

$\begin{array}{ll}- \text { Normal } & \nabla \text { Severe degradation } \\ \text { - Slight degradation } & \times \text { Center of K-Means }\end{array}$

(b) Results of LPP-based clustering

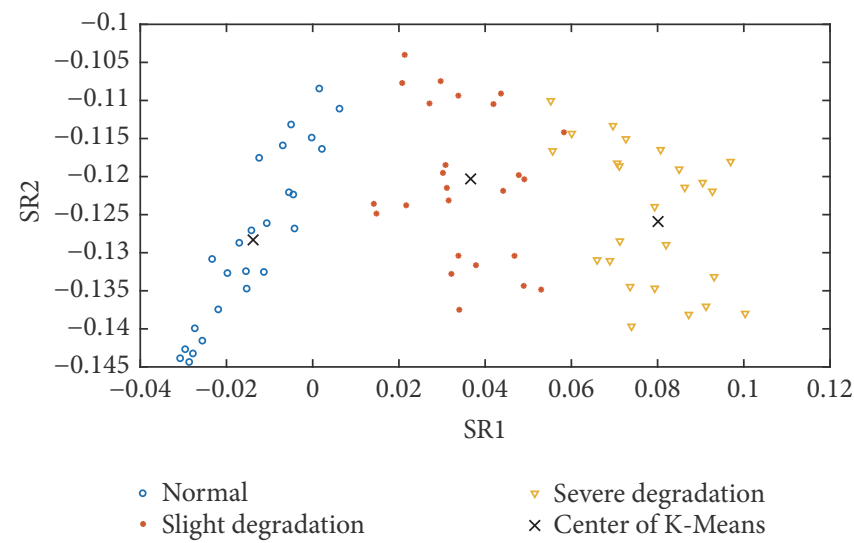

(c) Results of SR-based clustering

FIGURE 3: Clustering results of the three dimension reduction methods.

difference, and sample entropy tend to rise with fluctuations. Therefore, the extracted features could be used to characterize the performance of Lithium-ion batteries; however, these features have some drawbacks. Standard deviation, skewness, and kurtosis fluctuate violently and are not sensitive to the degradation of performance, making it difficult to determine the degree of degradation. It is observed that the standard deviation, skewness, kurtosis, and sample entropy of battery numbers 5 and 6 have a "run-in" period in the early stage, during which the values fluctuate violently without a significant trend. When the battery is diagnosed during this "run-in" period, it is hard to see a trend towards degradation.

To enable more accurate online monitoring of the battery degradation, this paper proposes a quantitative indicator obtained by fusing multiple multi-dimensional features to effectively describe battery degradation.

As soon as the battery is used, it begins to degrade. In order to reduce the error of the health state model, the first $4 \%$ of all cycles were selected for training. On the basis of the above analysis, the first $4 \%$ sets of health state data of battery numbers 5, 6, and 18 are chosen to establish the GMM model. A GMM with 2 Gaussian components is used to model the normal state data, and the first two dimensions after dimension reduction by SR are used as the input data. After the GMM model is trained with the normal state data, all of the test data is input into the model in groups, and one BID value is calculated using each data group, as shown in Figures 5, 6, and 7. Figure 5 shows that the BID value of battery number 5 effectively and clearly reflects the performance degradation process of the lithium-ion battery. Similarly, the trend towards battery degradation is reflected in Figures 6 and 7. The BID curve increases with the increase of the number of battery discharge cycles.

The assessment method based on SR-GMM-BID is superior to the methods that use only a single feature. First, BID value is sensitive to the performance degradation of the battery, and the trend of lithium-ion battery degradation can be clearly seen. Second, the fused indicator does not fluctuate violently in the early stage and improves the reliability of assessment.

\subsection{Comparative Analysis}

4.4.1. Comparison with the Assessment Method Based on the Support Vector Data Description (SVDD) Algorithm. The SVDD algorithm is an algorithm that is well-suited for assessing the performance of bearings [29]. In the experiment, the first $4 \%$ of all data groups were taken as the normal state data to train the SVDD model; the kernel function was defined as a Gaussian kernel, and the penalty factor 


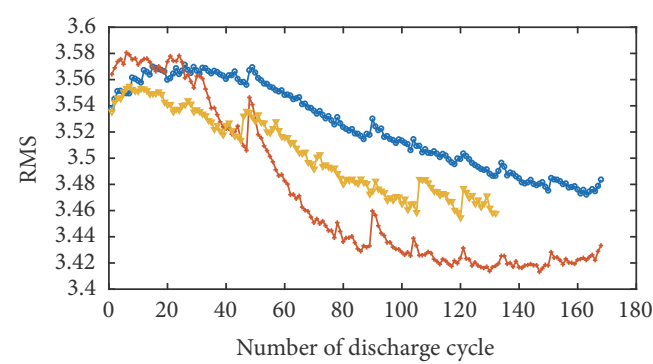

$\rightarrow$ Battery No. 5

- Battery No.6

- Battery No.18

(a) Variation of RMS values with an increase in the number of discharge cycles

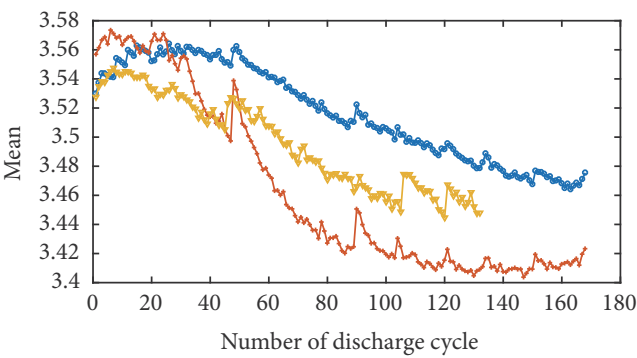

\section{$\rightarrow$ Battery No. 5 \\ + Battery No.6}

* Battery No.18

(c) Variation of mean values with an increase in the number of discharge cycles

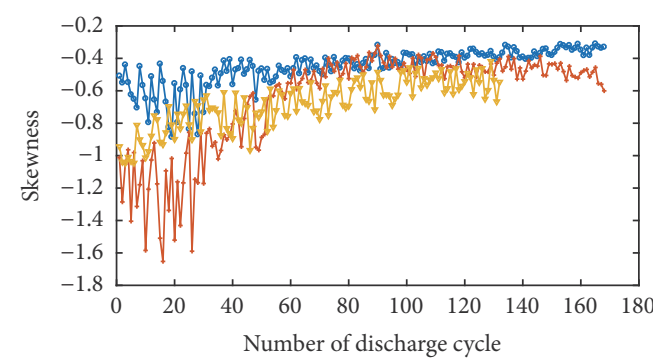

$$
\begin{aligned}
& \text { - Battery No.5 } \\
& \text { - Battery No.6 } \\
& \text { - Battery No. } 18
\end{aligned}
$$

(e) Variation of skewness values with an increase in the number of discharge cycles

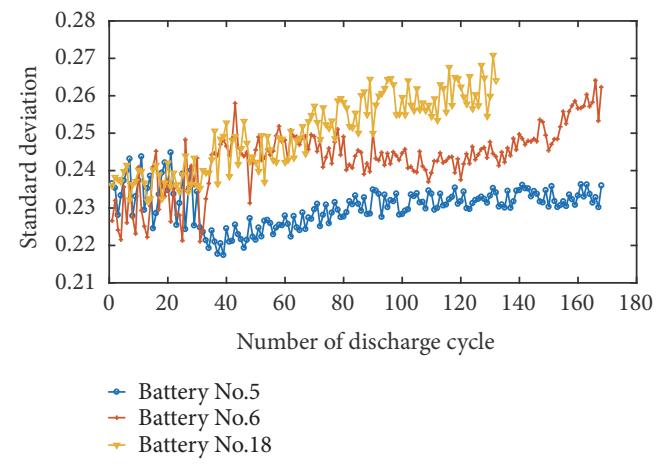

(b) Variation of standard deviation values with an increase in the number of discharge cycles

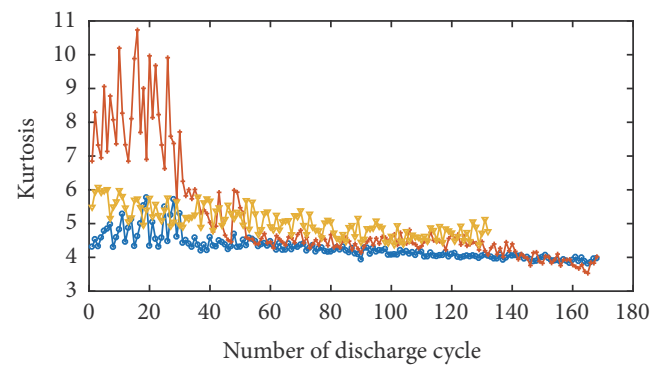

$$
\begin{aligned}
& \text { - Battery No.5 } \\
& \text { - Battery No.6 } \\
& \text { - Battery No.18 }
\end{aligned}
$$

(d) Variation of kurtosis values with an increase in the number of discharge cycles

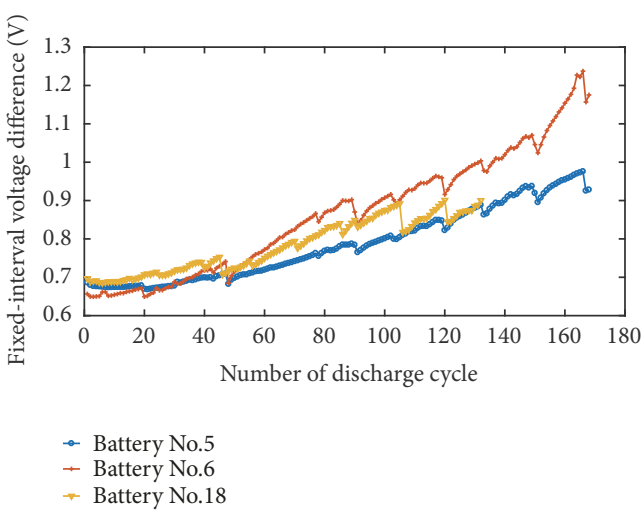

(f) Variation of fixed-interval voltage difference values with an increase in the number of discharge cycles

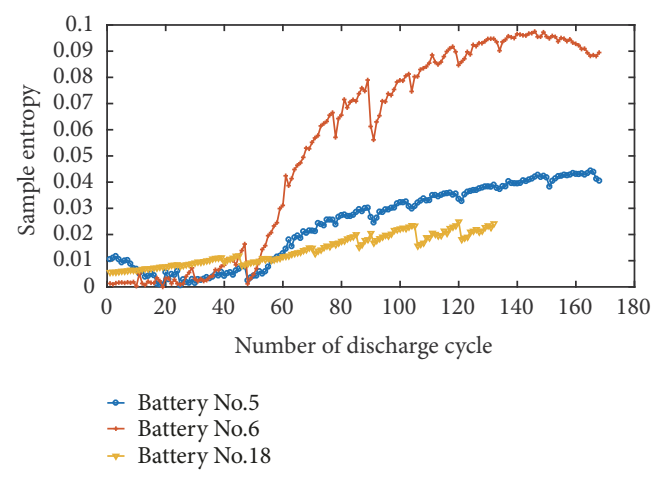

(g) Variation of sample entropy values with an increase in the number of discharge cycles

FIgURE 4: Variation trends of the 6 features exhibited by battery numbers 5, 6, and 18 . 


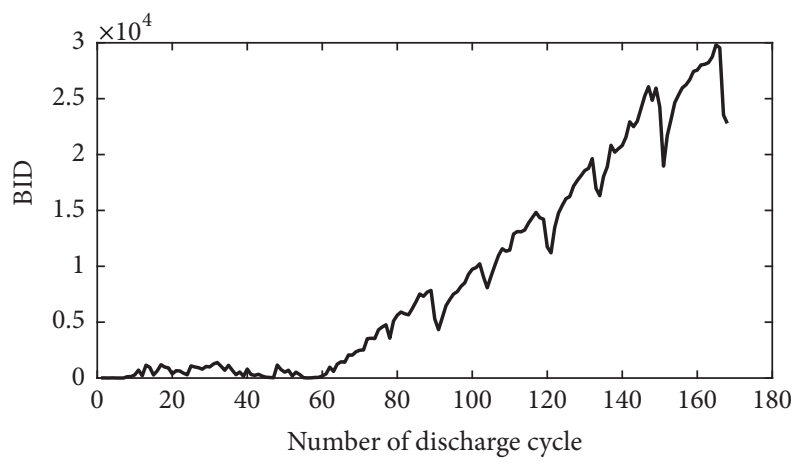

Figure 5: Assessing battery number 5 using BID values.

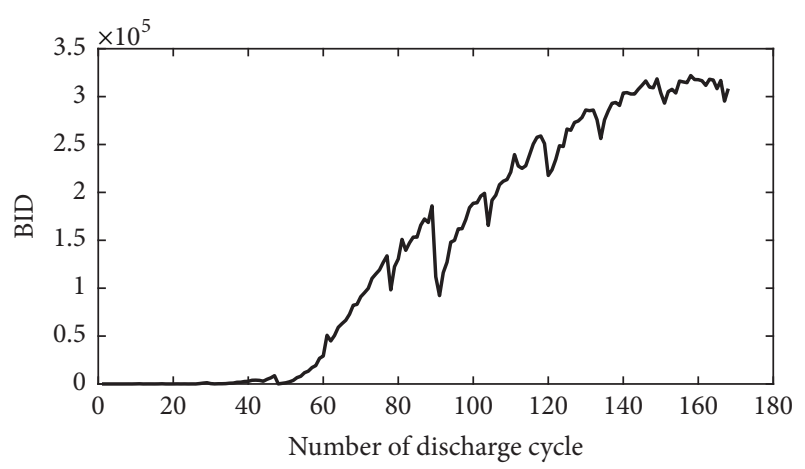

Figure 6: Assessing battery number 6 using BID values.

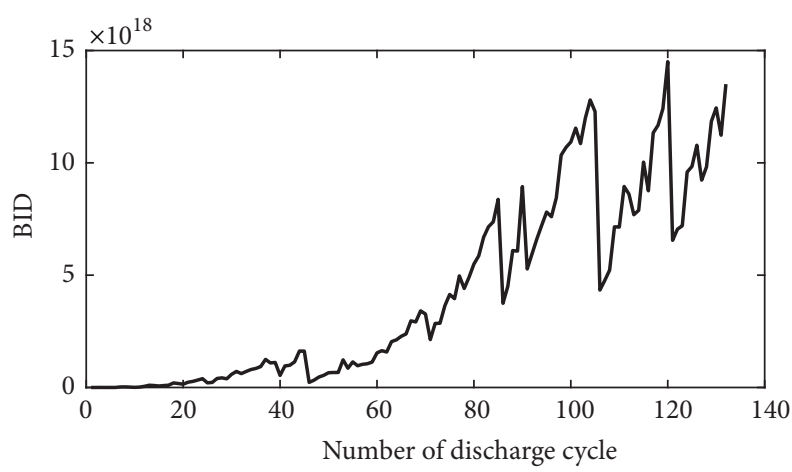

FIGURE 7: Assessing battery number 18 using BID values.

was set to 0.3. The value of the kernel distance between the test data and the trained hypersphere was used as the quantitative performance assessment parameter, and the assessment results based on SR-SVDD are shown in Figures 8,9 , and 10 .

4.4.2. Comparison with the Assessment Method Based on Another GMM Based Indicator. A negative logarithmic likelihood probability (NLLP) value was used to evaluate the performance of the bearing [30]; that is, after establishing the GMM model in normal state, a logarithmic likelihood value is obtained for each set of test data. The larger the value is, the farther the deviation is. In general, logarithmic likelihood values are less than 0 , so negative logarithmic likelihood values are used as assessment parameter, and the assessment

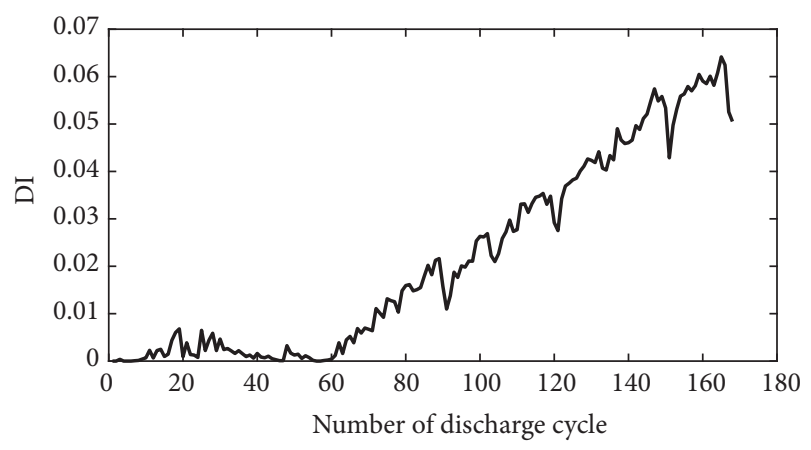

FIGURE 8: Assessing battery number 5 using the SVDD-based method.

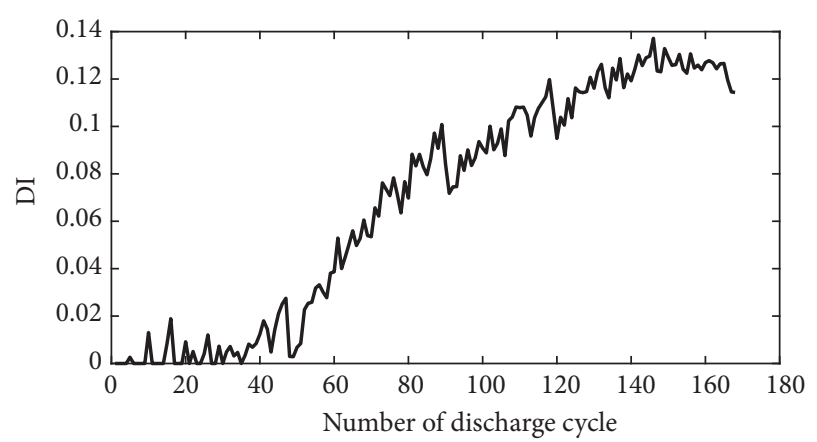

FIGURE 9: Assessing battery number 6 using the SVDD-based method.

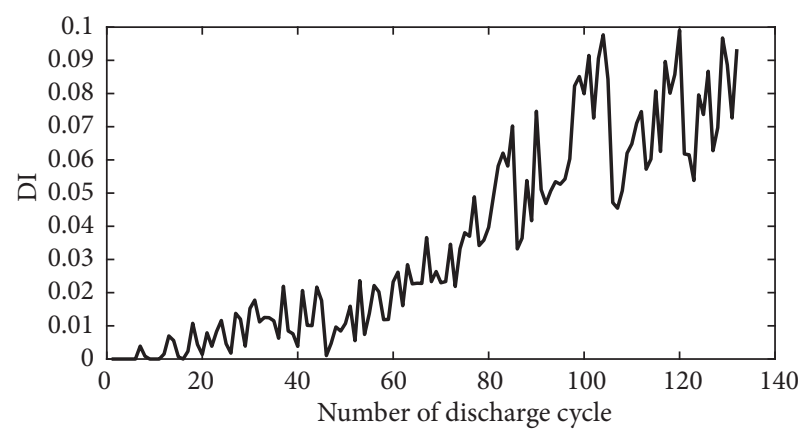

FIGURE 10: Assessing battery number 18 using the SVDD-based method.

results based on SR-GMM-NLLP are shown in Figures 11, 12, and 13.

4.4.3. Comparison with the Assessment Method Based on Self-Organizing Map(SOM). A data fusion method based on SOM is used to evaluate the bearing state [31]. SOM was used for network training, and multi-feature fusion model was constructed. After weight vector was obtained, the minimum quantization error (MQE) from each sample to the weight vector was calculated as the indicator, and the assessment results based on SOM are shown in Figures 14, 15, and 16.

4.4.4. The Results of Comparative Analysis. Spearman rank correlation coefficient is a typical analytical method to describe the linear relationship between two data sequences 
TABLE 3: Results of correlation analysis.

\begin{tabular}{lccc}
\hline & Battery No. 5 & Battery No. 6 & Battery No. 18 \\
\hline BID & -0.9969 & -0.9949 & -0.9926 \\
DI & -0.9926 & -0.9627 & -0.9105 \\
NLLP & -0.9941 & -0.9790 & -0.9418 \\
MQE & -0.9927 & -0.9483 & -0.9288 \\
\hline
\end{tabular}

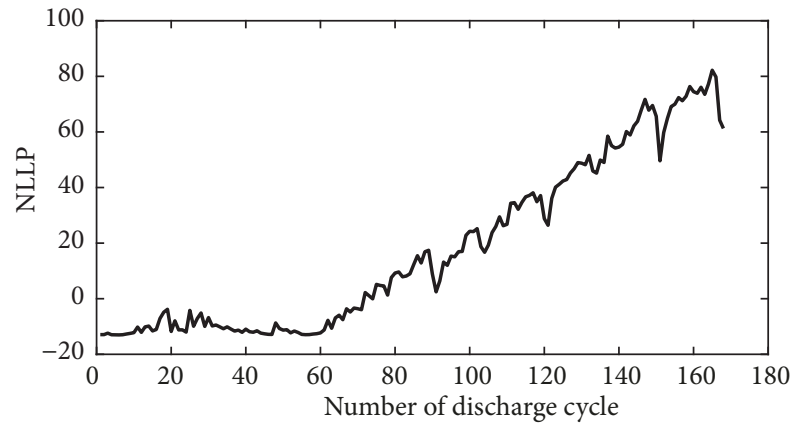

FIgURE 11: Assessing battery number 5 using the NLLP-based method.

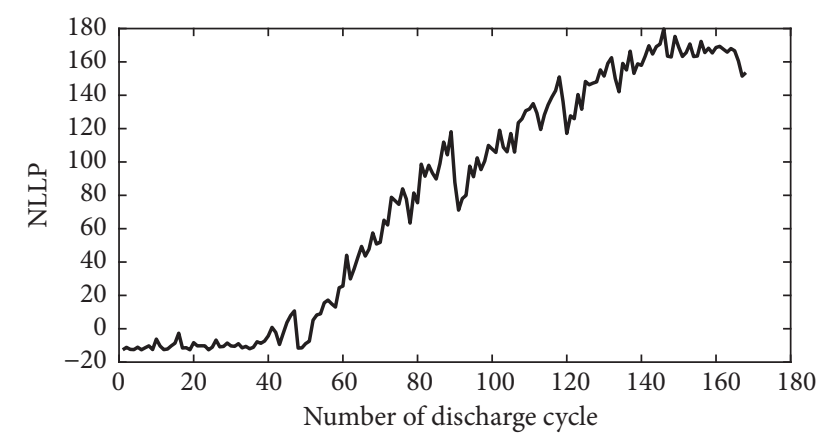

FIgURE 12: Assessing battery number 6 using the NLLP-based method.

[32]. In order to prove the superiority of the method proposed in this paper, the four fusion parameters and battery capacity were, respectively, analyzed by Spearman correlation. The interval of coefficient $r$ is $[-1,1]$. When $r$ is greater than 0 , it means positive correlation; when $r$ is less than 0 , it means negative correlation. The closer the absolute value of $r$ is to 1 , the more linearly dependent it is.

The correlation analysis results are shown in Table 3. The four health indicators are negative correlated with battery capacity. The absolute value of correlation coefficient of BID is significantly higher than DI, NLLP, and MQE. It indicates that the BID index has the best correlation with the battery capacity and can be used for the input of battery remaining life prediction (RUL) to improve the prognosis accuracy.

\section{Conclusions}

This paper proposes a model based on fusion of multiple features for assessing performance degradation of lithiumion batteries. The study yielded the following findings:

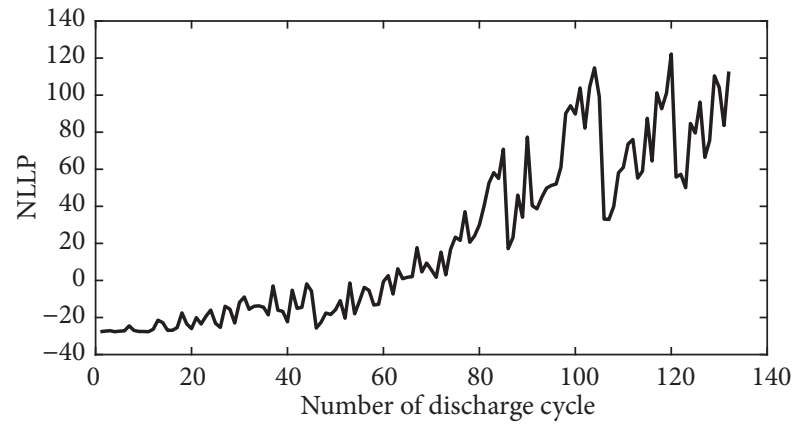

FIGURE 13: Assessing battery number 18 using the NLLP-based method.

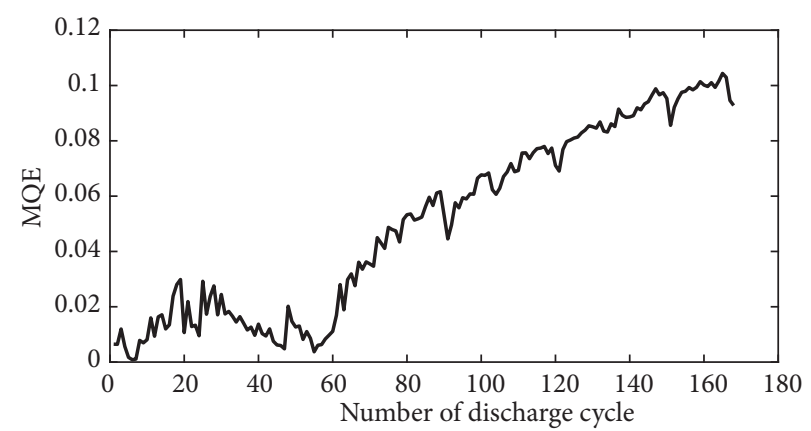

FIgURE 14: Assessing battery number 5 using the SOM-based method.

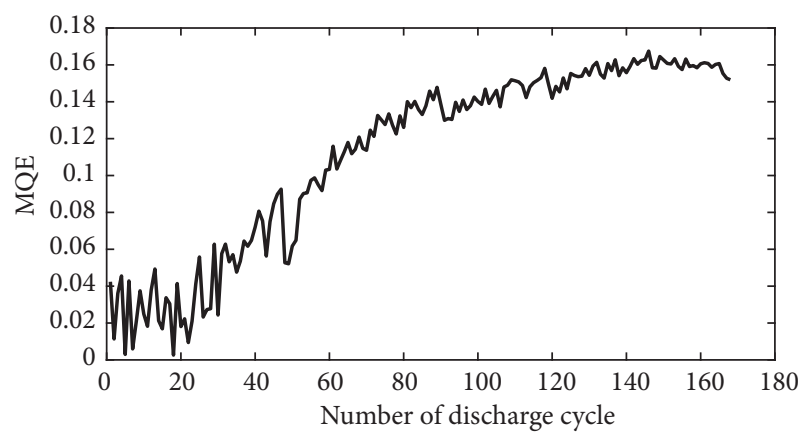

FIGURE 15: Assessing battery number 6 using the SOM-based method.

(1) The method based on fusion of multiple features proposed in this paper can be used to conduct effective assessment of the performance degradation of lithium-ion batteries.

(2) Compared with the PCA and LPP methods, the dimension reduction method based on SR can better cluster 


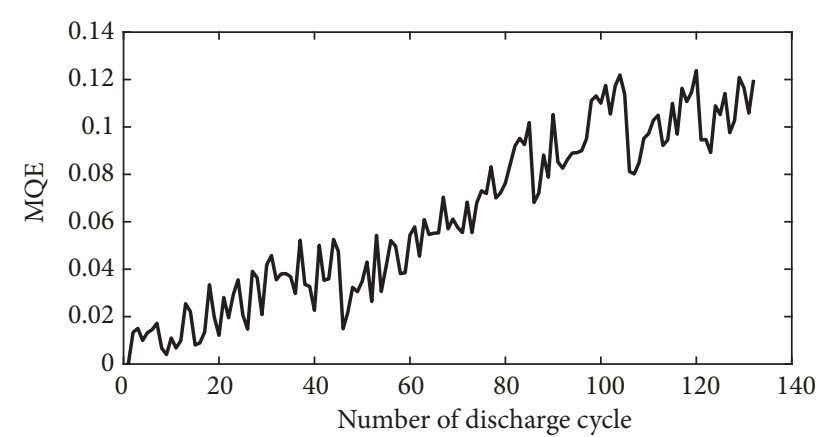

Figure 16: Assessing battery number 18 using the SOM-based method.

the measurement data into different degradation stages for lithium-ion batteries.

(3) The SR-GMM-BID-based quantitative performance assessment method proposed in this paper can better characterize the degradation trend of batteries than the SVDD, NLLP, and SOM based methods.

\section{Data Availability}

The data used to support the findings of this study are included within the article.

\section{Conflicts of Interest}

The authors declare no conflicts of interest. The funders had no role in the design of the study; in the collection, analyses, or interpretation of data; in the writing of the manuscript, or in the decision to publish the results.

\section{Authors' Contributions}

Anchen Wang and Ying Zhang contributed equally to this work. Conceptualization is done by Anchen Wang, Ying Zhang, and Hongfu Zuo; Data curation is done by Anchen Wang and Ying Zhang; Formal analysis is done by Anchen Wang and Ying Zhang; Methodology is done by Anchen Wang, Ying Zhang, and Hongfu Zuo; Software is done by Anchen Wang and Ying Zhang; Validation is done by Hongfu Zuo; Writing - original draft is done by Anchen Wang; Writing - review \& editing are done by Ying Zhang and Hongfu Zuo.

\section{Acknowledgments}

This research was supported by National Natural Science Foundation of China, grant number 51505229.

\section{References}

[1] J. Zhang and J. Lee, "A review on prognostics and health monitoring of Li-ion battery," Journal of Power Sources, vol. 196, no. 15, pp. 6007-6014, 2011.
[2] Y. Zou, X. Hu, H. Ma, and S. E. Li, "Combined State of Charge and State of Health estimation over lithium-ion battery cell cycle lifespan for electric vehicles," Journal of Power Sources, vol. 273, pp. 793-803, 2015.

[3] Y. Ma, Y. Chen, X. Zhou, and H. Chen, "Remaining useful life prediction of lithium-ion battery based on gauss-hermite particle filter," IEEE Transactions on Control Systems Technology, vol. 3, pp. 1-8, 2018.

[4] F. Yang, D. Wang, Y. Xing, and K.-L. Tsui, "Prognostics of $\mathrm{Li}(\mathrm{NiMnCo}) \mathrm{O} 2$-based lithium-ion batteries using a novel battery degradation model," Microelectronics Reliability, vol. 70, pp. 70-78, 2017.

[5] M. A. Hannan, M. S. H. Lipu, A. Hussain, and A. Mohamed, "A review of lithium-ion battery state of charge estimation and management system in electric vehicle applications: Challenges and recommendations," Renewable \& Sustainable Energy Reviews, vol. 78, pp. 834-854, 2017.

[6] C. Sbarufatti, M. Corbetta, M. Giglio, and F. Cadini, "Adaptive prognosis of lithium-ion batteries based on the combination of particle filters and radial basis function neural networks," Journal of Power Sources, vol. 344, pp. 128-140, 2017.

[7] X. Xu and N. Chen, "A state-space-based prognostics model for lithium-ion battery degradation," Reliability Engineering \& System Safety, vol. 159, pp. 47-57, 2017.

[8] X. Xu, Z. Li, and N. Chen, "A hierarchical model for lithiumion battery degradation prediction," IEEE Transactions on Reliability, vol. 65, no. 1, pp. 310-325, 2016.

[9] A. Widodo, M.-C. Shim, W. Caesarendra, and B.-S. Yang, "Intelligent prognostics for battery health monitoring based on sample entropy," Expert Systems with Applications, vol. 38, no. 9, pp. 11763-11769, 2011.

[10] S. Dong and T. Luo, "Bearing degradation process prediction based on the PCA and optimized LS-SVM model," Measurement, vol. 46, no. 9, pp. 3143-3152, 2013.

[11] J.-B. Yu, "Bearing performance degradation assessment using locality preserving projections," Expert Systems with Applications, vol. 38, no. 6, pp. 7440-7450, 2011.

[12] D. Cai, X. He, and J. Han, "Spectral regression:a unified approach for sparse subspace learning," in IEEE International Conference on Data Mining, pp. 73-82, IEEE, 2007.

[13] Z. Chen and W. Li, "Multisensor feature fusion for bearing fault diagnosis using sparse autoencoder and deep belief network," IEEE Transactions on Instrumentation and Measurement, vol. 66, no. 7, pp. 1693-1702, 2017.

[14] L. Batista, B. Badri, R. Sabourin, and M. Thomas, "A classifier fusion system for bearing fault diagnosis," Expert Systems with Applications, vol. 40, no. 17, pp. 6788-6797, 2013.

[15] Y. T. Ai, J. Y. Guan, W. C. Fei et al., "Fusion information entropy method of rolling bearing fault diagnosis based on n -dimensional characteristic parameter distance," Mechanical Systems \& Signal Processing, vol. 88, pp. 123-136, 2017.

[16] H. Hassani, J. Zarei, M. M. Arefi, and R. Razavi-Far, “ZSlicesbased general type-2 fuzzy fusion of support vector machines with application to bearing fault detection," IEEE Transactions on Industrial Electronics, vol. 64, no. 9, pp. 7210-7217, 2017.

[17] B. Zhang, L. Zhang, J. Xu, and P. Wang, "Performance degradation assessment of rolling element bearings based on an index combining SVD and information exergy," Entropy, vol. 16, no. 10, pp. 5400-5415, 2014.

[18] H. Shao, H. Jiang, X. Zhang, and M. Niu, "Rolling bearing fault diagnosis using an optimization deep belief network," 
Measurement Science and Technology, vol. 26, no. 11, Article ID $115002,2015$.

[19] H. T. Zhou, J. Chen, G. M. Dong, H. C. Wang, and H. D. Yuan, "Bearing fault recognition method based on neighbourhood component analysis and coupled hidden Markov model," Mechanical Systems and Signal Processing, Article ID S0888327015002204, 2015.

[20] D. Wang, K.-L. Tsui, and Q. Miao, "Prognostics and health management: a review of vibration based bearing and gear health indicators," IEEE Access, vol. 6, pp. 665-676, 2017.

[21] D. H. Liu, H. Wang, Y. Peng, W. Xie, and H. T. Liao, "Satellite lithium-ion battery remaining cycle life prediction with novel indirect health indicator extraction," Energies, vol. 6, no. 8, pp. 3654-3668, 2013.

[22] A. K. Charakopoulos, T. E. Karakasidis, P. N. Papanicolaou, and A. Liakopoulos, "Nonlinear time series analysis and clustering for jet axis identification in vertical turbulent heated jets," Physical Review E: Statistical, Nonlinear, and Soft Matter Physics, vol. 89, no. 3, Article ID 032913, 2014.

[23] M. Han and J. Pan, "A fault diagnosis method combined with LMD, sample entropy and energy ratio for roller bearings," Measurement, vol. 76, pp. 7-19, 2015.

[24] M. Seera, M. L. D. Wong, and A. K. Nandi, "Classification of ball bearing faults using a hybrid intelligent model," Applied Soft Computing, vol. 57, pp. 427-435, 2017.

[25] Y. Gao, M. Karimi, A. A. Kudreyko, and W. Song, "Spare optimistic based on improved ADMM and the minimum entropy de-convolution for the early weak fault diagnosis of bearings in marine systems," ISA Transactions, Article ID S0019057817306377, 2017.

[26] C. Federico, "A review of data fusion techniques," The Scientific World Journal, vol. 2013, Article ID 704504, 19 pages, 2013.

[27] X. Xie and H. Shi, "Global monitoring strategy for multimode chemical processes," Journal of Chemical Industry and Engineering(China), vol. 63, no. 7, pp. 2156-2162, 2012.

[28] B. Saha and K. Goebel, Battery Data Set, NASA Ames Prognostics Data Repository, NASA Ames, Moffett Field, Calif, USA, 2007, https:/ti.arc.nasa.gov/tech/dash/groups/pcoe/prognostic-datarepository/\#battery.

[29] Y. N. Pan, J. Chen, and L. Guo, "Robust bearing performance degradation assessment method based on improved wavelet packet-support vector data description," Mechanical Systems and Signal Processing, vol. 23, no. 3, pp. 669-681, 2009.

[30] J. Yu, "Bearing performance degradation assessment using locality preserving projections and Gaussian mixture models," Mechanical Systems and Signal Processing, vol. 25, no. 7, pp. 2573-2588, 2011.

[31] R. Huang, L. Xi, X. Li, C. Richard Liu, H. Qiu, and J. Lee, "Residual life predictions for ball bearings based on selforganizing map and back propagation neural network methods," Mechanical Systems and Signal Processing, vol. 21, no. 1, pp. 193-207, 2007.

[32] C. Spearman, "The proof and measurement of association between two things," The American Journal of Psychology, vol. 100, no. 3-4, pp. 441-471, 1987. 


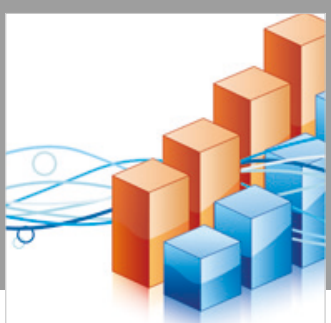

Advances in

Operations Research

\section{-n-m}
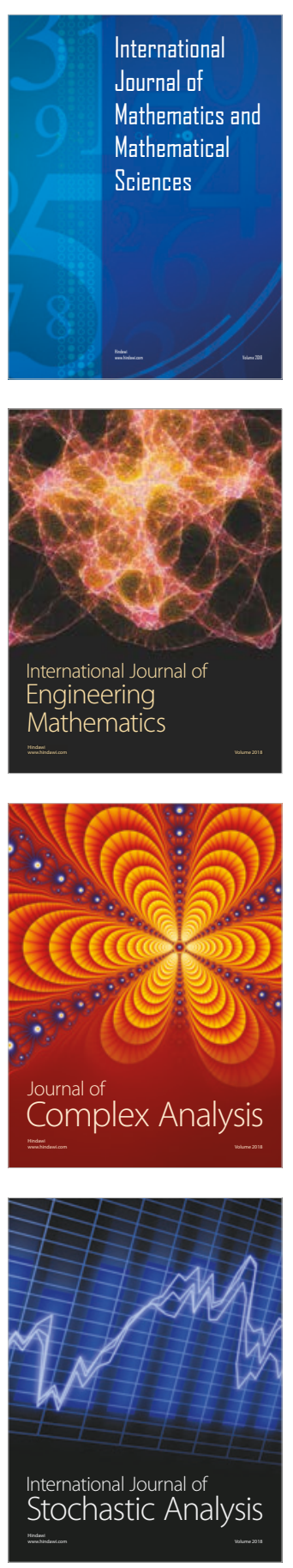
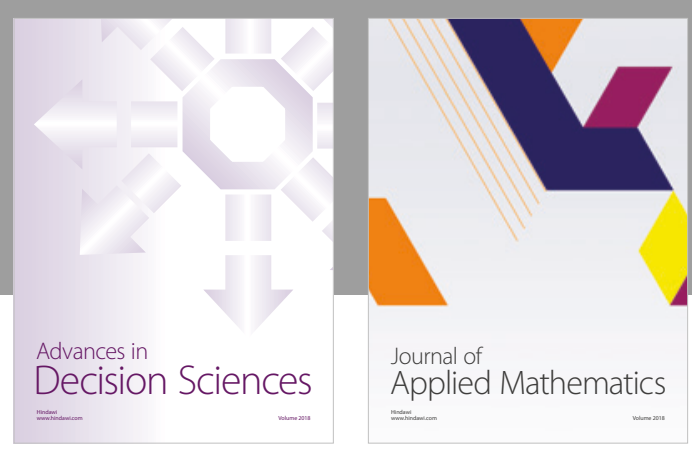

Journal of

Applied Mathematics
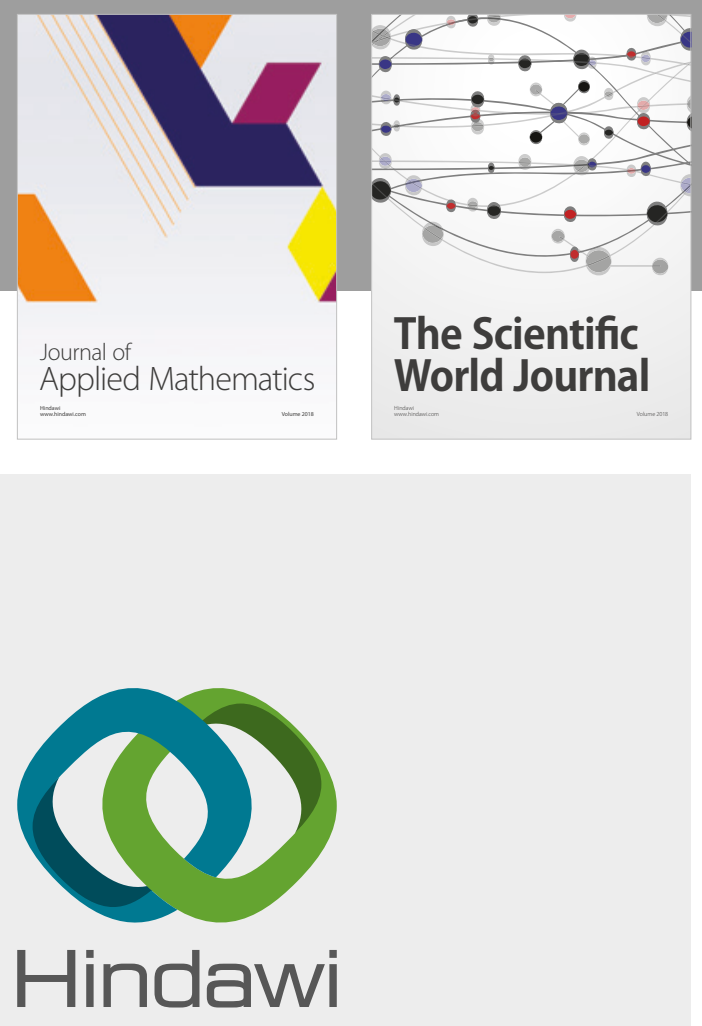

Submit your manuscripts at

www.hindawi.com

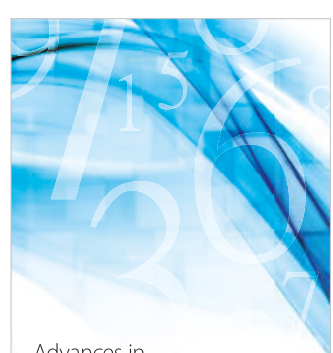

Advances in
Numerical Analysis
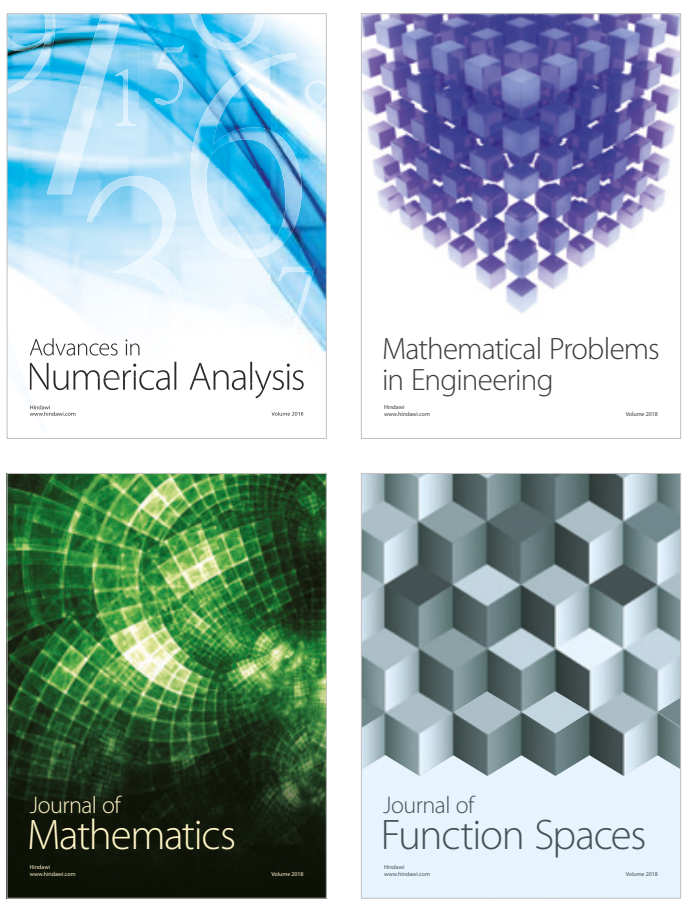

Mathematical Problems in Engineering

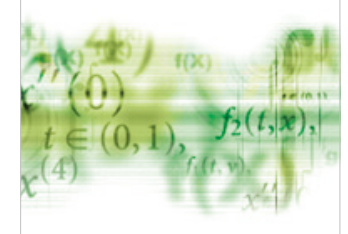

International Journal of

Differential Equations

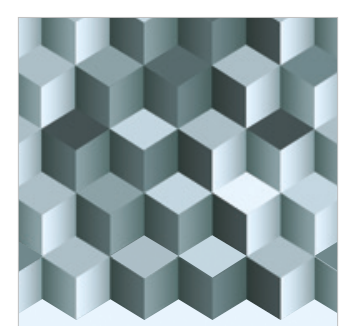

Journal of

Function Spaces

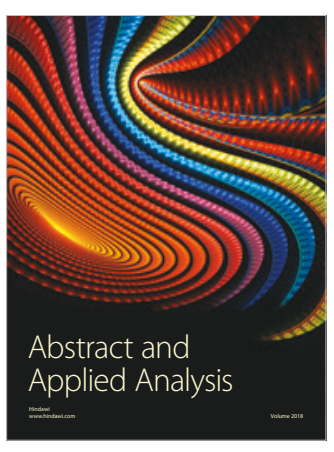

The Scientific

World Journal

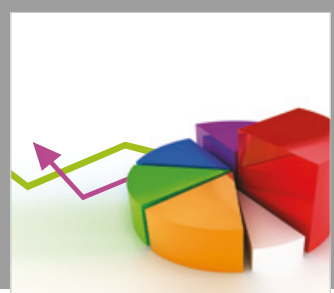

Journal of

Probability and Statistics
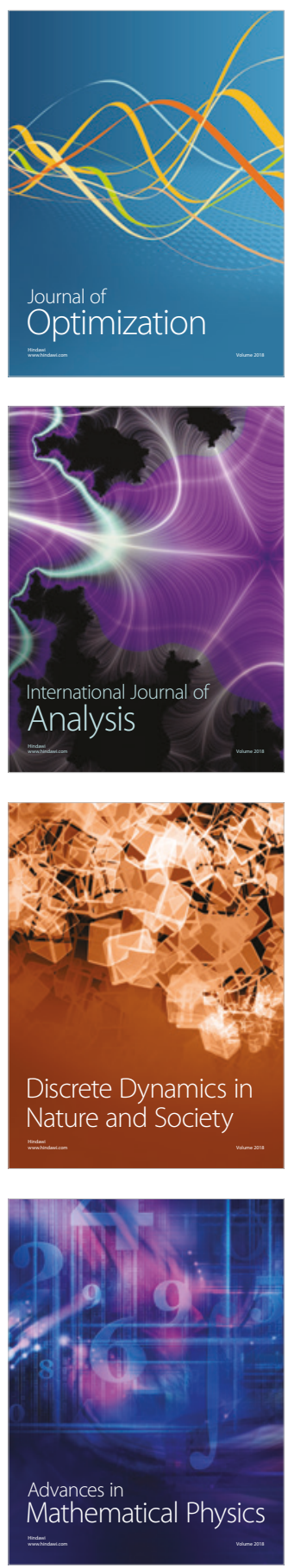